\title{
Seroepidemiological Survey of Pathogenic Yersinia in Breeding Squirrel Monkeys in Japan
}

\author{
Taketoshi IWATA ${ }^{1)}$, Yumi UNE²), Ken-ichi LEE ${ }^{1)}$, Shin-ichi NAKAMURA ${ }^{2)}$, Takahide TANIGUCHI ${ }^{1)}$ and \\ Hideki HAYASHIDANI ${ }^{1) *}$ \\ ${ }^{1)}$ Division of Animal Life Science, Institute of Agriculture, Tokyo University of Agriculture and Technology, 3-5-8 Saiwai-cho, Fuchu, \\ Tokyo 183-8509 and ${ }^{2}$ School of Veterinary Medicine, Azabu University, 1-17-71 Fuchinobe, Sagamihara, Kanagawa 229-8501, Japan
}

(Received 10 November 2009/Accepted 2 March 2010/Published online in J-STAGE 16 March 2010)

ABSTRACT. To investigate the prevalence of antibodies to pathogenic Yersinia in breeding squirrel monkeys, the serum samples of 252 squirrel monkeys from 9 zoological gardens in Japan were tested by ELISA using plasmid-encoded Yersinia outer membrane protein (Yops) as the antigen. The cutoff value was calculated by using the serum samples of the squirrel monkeys from Suriname, where no prevalence of pathogenic Yersinia have been reported. According to the cutoff value, 164 of 252 (65.1\%) squirrel monkeys were considered positive against pathogenic Yersinia. These positive monkeys belonged to 8 of the 9 zoological gardens, and the percentage of the seropositive monkeys ranged from 22.2 to $89.4 \%$. Furthermore, in one zoological garden, the positive rate of the squirrel monkeys which were over 1 year old $(95.7 \%)$ was significantly higher than those which were under 1 year old (23.3\%). These results suggested that pathogenic Yersinia is highly prevalent among breeding monkeys in Japan.

KEY WORDS: ELISA, squirrel monkey, Yersinia, Yops.

J. Vet. Med. Sci. 72(8): 981-984, 2010

Yersiniosis is an infection with pathogenic Yersinia, which is comprised of pathogenic $Y$. enterocolitica and $Y$. pseudotuberculosis. These pathogens cause gastrointestinal symptoms including enteritis, diarrhea and mesenteric lymphadenitis, and sometimes septicemia in humans and animals $[3,18]$. Monkey species are especially sensitive to yersiniosis, and many fatal cases in breeding monkeys have been reported throughout the world [2, 8, 17, 23, 27]. In Japan, $Y$. pseudotuberculosis in particular frequently causes fatal infection in breeding monkeys $[11,14,15,30]$. The highest number of dead monkeys by $Y$. pseudotuberculosis infection in Japan has occurred among the squirrel monkey (Saimiri spp.) [14, 30]. The habitat of the squirrel monkey is South and Central America, but many zoological gardens in Japan have been breeding monkeys imported from those regions. Many authors have published clinical and/or pathological reports of fatal infection with pathogenic Yersinia in breeding monkeys, including squirrel monkeys, but detailed information on the epidemiology of yersiniosis in breeding monkeys has not yet been obtained.

The pathogenicity of pathogenic strains of Yersinia depends on the presence of a $70-\mathrm{kb}$ virulence plasmid termed "pYV". This plasmid is essential for virulence and is used to differentiate pathogenic from nonpathogenic Yersinia. To establish infection and subvert host defenses, pathogenic Yersinia require a type III secretion system which translocates virulence factors, called Yops (Yersinia outer membrane proteins), into host cells $[6,25]$. Some researchers have reported that enzyme-linked immunosor-

\footnotetext{
* Correspondence to: Hayashidani, H., Division of Animal Life Science, Institute of Agriculture, Tokyo University of Agriculture and Technology, 3-5-8 Saiwai-cho, Fuchu, Tokyo 1838509, Japan.

e-mail: eisei@cc.tuat.ac.jp
}

bent assay (ELISA) and immunoblot assays using Yops as antigen are a specific and sensitive method for detecting pathogenic Yersinia infection [22, 26, 28]. To determine the prevalence of pathogenic Yersinia infection in breeding monkeys, we conducted a seroepidemiological study in squirrel monkeys in Japan by ELISA using semi-purification Yops as antigen.

\section{MATERIALS AND METHODS}

Serum samples: Two hundreds fifty-two serum samples were collected from 9 zoological gardens (A-I) in Japan, and tested by ELISA for antibodies to Yops. In addition, 91 serum samples which were collected from Suriname immediately after importation were used as negative control. The serum samples were stored at $-20^{\circ} \mathrm{C}$ until use, and inactivated at $56^{\circ} \mathrm{C}$ for $30 \mathrm{~min}$ before use.

Yops preparation: Yops were prepared according to the method of Heesemann et al. [12]. Y. pseudotuberculosis serovar $4 \mathrm{~b}$ isolated from a dead squirrel monkey was precultured in BHI broth (Becton, Dickinson and Company, Franklin Lakes, NJ, U.S.A.) at $25^{\circ} \mathrm{C}$ with shaking (110 rpm) overnight. This preculture was then diluted 1:20 with fresh BHI broth and incubated with shaking $(110 \mathrm{rpm})$ at $37^{\circ} \mathrm{C}$ for 90 min. Filter-sterilized EGTA (Sigma, St. Louis, MO, U.S.A.) was added to the medium to final concentration of $2.5 \mathrm{mM}$, and incubation was continued for $90 \mathrm{~min}$ at $37^{\circ} \mathrm{C}$. The bacterial cells were then removed by centrifugation $\left(7,000 \times g\right.$ at $4^{\circ} \mathrm{C}$ for $\left.20 \mathrm{~min}\right)$, and clarified culture supernatant was filter-sterilized. The proteins were precipitated from this culture supernatant by the addition of solid ammonium sulfate $(40 \mathrm{~g} / 100 \mathrm{~m} l$ of supernatant $)$. The precipitated proteins were dissolved in distilled water, and dialyzed with 
Spectra/PoraCE Membrane MWCO:10,000 (Spectrum ${ }^{\circledR}$ Laboratories Inc., Rancho Dominguez, CA, U.S.A.). The retained volume was lyophilized and stored at $-30^{\circ} \mathrm{C}$ until use.

SDS-PAGE: Yops were examined by sodium dodecyl sulfate-polyacrylamide gel electrophoresis (SDS-PAGE). The method used for SDS-PAGE was essentially the one described by Laemmli [16]. Briefly, Yops was suspended in Laemmli sample buffer (Bio-Rad Laboratories, Inc., Hercules, CA, U.S.A.), boiled for $3 \mathrm{~min}$, and then subjected to a $12.0 \%$ polyacrylamide gel. After that, the gel was stained with silver nitrate.

ELISA: ELISA was carried out in flat-bottom 96-well microtiter plates (MaxiSorp; Nunc, Roskilde, Denmark). The plates were coated with $250 \mu \mathrm{g}$ of Yops antigen $/ \mathrm{ml}$ (50 $\mu l /$ well) in phosphate buffer saline (pH 7.2) and incubated overnight at $4{ }^{\circ} \mathrm{C}$. The wells were then blocked with Diluent/Blocking Concentrate (Kirkegaard and Perry Laboratories, Inc., Gaithersburg, MD, U.S.A.) at $25^{\circ} \mathrm{C}$ for $15 \mathrm{~min}$. In each of the three wells assigned for the individual serum sample, the wells were loaded with the sample (1:40 dilution in Wash solution; KPL) and incubated at $37^{\circ} \mathrm{C}$ for $1 \mathrm{hr}$. The plates were washed three times with Wash solution and incubated with peroxidase-conjugated Protein G (1:1,000 dilution in Diluent/Blocking Concentrate; Invitrogen Co., Carlsbad, CA, U.S.A.) at $25^{\circ} \mathrm{C}$ for $1 \mathrm{hr}$. After being washed five times, the plates were incubated with substrate ABTS (KPL) for $20 \mathrm{~min}$ at $25^{\circ} \mathrm{C}$, and the optical density (OD) was measured at $405 \mathrm{~nm}$ by a MTP-120 microplate reader (Corona Electric Co. Ltd., Ibaraki, Japan).

Cutoff value: The OD values of 91 monkeys from Suriname, where no presence of pathogenic Yersinia have been reported [9], were considered to be a negative control. The cutoff value was calculated as the mean OD of the negative sera plus 3 standard deviations (SD). The Yops antibodies were considered positive when the OD value was higher than the cutoff value. The OD values of the 91 monkeys from Suriname ranged between 0.023 and 0.112 , and the mean was 0.050 (Fig. 2). The SD was calculated to be 0.021 from those results. Therefore, the cutoff value was calculated to be 0.113 .

\section{RESULTS}

SDS-PAGE analysis of Yops: The silver stained Yops showed 5 bands, and low background (Fig. 1). Designated bands (A-E) were considered to be YopH (51.0 kDa), YopB (41.8 kDa), YopD (33.3 kDa), YopN (32.6 kDa) and YopE $(22.9 \mathrm{kDa})$, respectively $[6,21]$.

Prevalence of $\operatorname{Ig} G$ antibodies to Yops in squirrel monkeys in Japan: Among the 252 squirrel monkeys tested, 164 (65.1\%) showed an OD higher than the cutoff value, 0.113 , and were therefore considered positive (Fig. 2). These positive monkeys belonged to 8 of the 9 zoological gardens, and the percentage of the seropositive monkeys ranged from 22.2 to $89.4 \%$ (Table 1 ).

Prevalence of serum antibody to Yops by age in squirrel

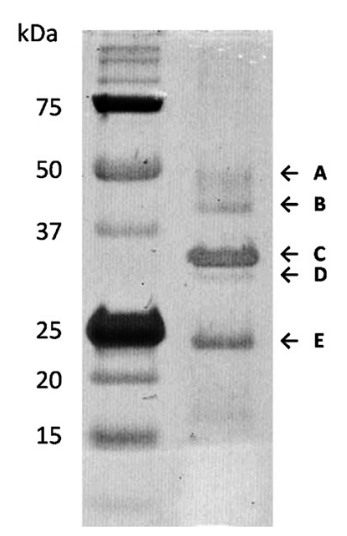

Fig. 1. SDS-PAGE followed by silver staining of the Yops. Left lane shows the prestained broad range protein molecular mass markers (Bio-Rad), and right lane shows Yops used as the antigen for ELISA.

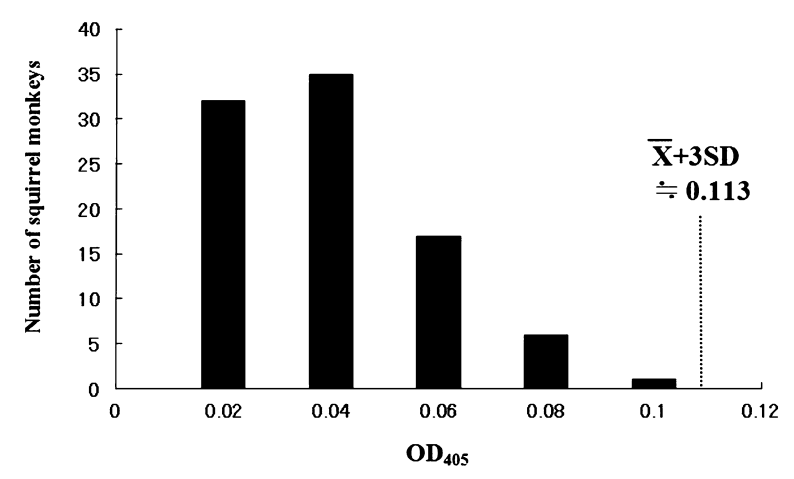

Fig. 2. Antibody titers to Yops of 91 squirrel monkeys just after imported from Suriname. The vertical dashed line represents the cutoff value, which was calculated as 3 standard deviations (SD) from the mean of this group.

monkeys of institution $H$ : All squirrel monkeys in institution $\mathrm{H}$ were individually recognized by electronic microchips, so that information about them, including the age, was controlled. To investigate the relationship between the age and prevalence of pathogenic Yersinia, the prevalence of antibody to Yops in institution $\mathrm{H}$ was arranged by age (Table 2). The positive rate of the monkeys that were over 1 year old $(95.7 \%)$ was significantly higher than that under 1 year old $(23.3 \%)(P<0.05)$.

\section{DISCUSSION}

The present study demonstrated that pathogenic Yersinia is highly prevalent among breeding monkeys in Japan. Yops used as an antigen of ELISA are encoded in pYV, which is harbored in pathogenic strains of Yersinia. Regardless of the species and serovars of Yersinia, it is known that pathogenic Yersinia infection elicits specific antibody response to Yops in humans and animals [5, 13, 19]. Therefore, the squirrel monkeys considered Yops positive in the 


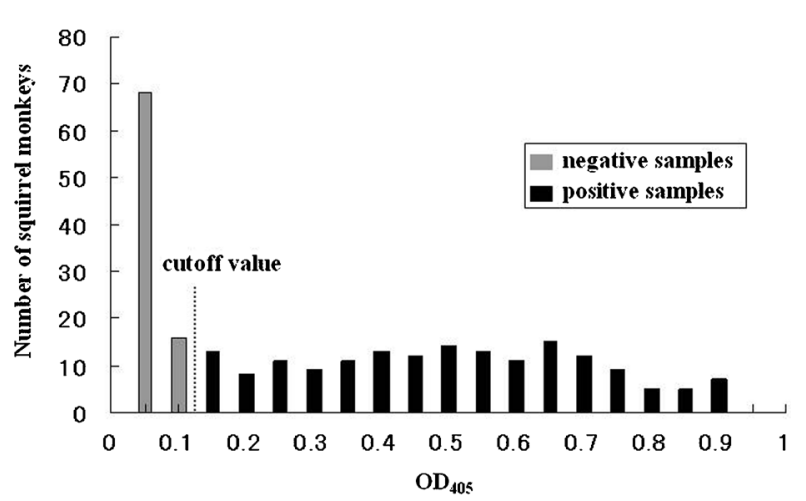

Fig. 3. Analysis of sera obtained from 252 breeding squirrel monkeys in Japan. The vertical dashed line represents the cutoff value, which was calculated as 0.113 .

Table 1. Prevalence of serum antibody to Yops in squirrel monkeys from 9 institutions in Japan

\begin{tabular}{lccc}
\hline Region & Institution & \multicolumn{2}{c}{$\begin{array}{c}\text { No. of positive samples/ } \\
\text { No. of samples tested } \\
\text { (\%) }\end{array}$} \\
\hline Kanto & $\mathrm{A}$ & $9 / 23$ & $(39.1)$ \\
& $\mathrm{B}$ & $12 / 15$ & $(80.0)$ \\
& $\mathrm{C}$ & $0 / 10$ & $(0.0)$ \\
\multirow{3}{*}{ Kinki } & $\mathrm{D}$ & $2 / 9$ & $(22.2)$ \\
& $\mathrm{E}$ & $6 / 8$ & $(75.0)$ \\
& $\mathrm{F}$ & $6 / 23$ & $(26.1)$ \\
\hline Shikoku & $\mathrm{G}$ & $11 / 14$ & $(78.6)$ \\
Kyusyu & $\mathrm{H}$ & $76 / 103$ & $(73.8)$ \\
& $\mathrm{I}$ & $42 / 47$ & $(89.4)$ \\
\hline Total & & $164 / 252$ & $(65.1)$ \\
\hline
\end{tabular}

Table 2. Prevalence of serum antibody to Yops by age in squirrel monkeys of institution $\mathrm{H}$

\begin{tabular}{cccc}
\hline Age (years) & $\begin{array}{c}\text { No. of positive samples / } \\
\text { No. of samples tested } \\
(\%)\end{array}$ & $\begin{array}{c}\text { Average } \\
\text { OD value }\end{array}$ \\
\hline$<1$ & $7 / 30$ & $(23.3)$ & 0.084 \\
$1-2$ & $13 / 15$ & $(86.7)$ & 0.300 \\
$2-3$ & $18 / 18$ & $(100.0)$ & 0.420 \\
$3-4$ & $3 / 4$ & $(75.0)$ & 0.389 \\
$4-5$ & $10 / 10$ & $(100.0)$ & 0.494 \\
$5-6$ & $7 / 7$ & $(100.0)$ & 0.559 \\
$>6$ & $16 / 16$ & $(100.0)$ & 0.420 \\
Unknown & $2 / 3$ & $(66.7)$ & 0.261 \\
\hline Total & $76 / 103$ & $(73.8)$ & 0.315 \\
\hline
\end{tabular}

present study must have been infected by pathogenic Yersinia in the past. However, squirrel monkeys that do not have any immunity to yersiniosis, such as infant monkeys, seem to die at a high rate when infected with $Y$. pseudotuberculosis considering past studies [14, 30].

Pathogenic Yersinia can be divided into low pathogenic strains, which induce a mild intestinal infection in humans, and highly pathogenic strains, which cause severe systemic infection in humans $[4,10]$. Whether pathogenic Yersinia causes limited gastroenteritis or systemic infection in humans correlate with the presence of a high-pathogenicity island (HPI), encoding an iron uptake system represented by its siderophore yersiniabactin [4] or Y. pseudotuberculosisderived mitogen (YPM), which is a superantigenic toxin [1]. It is known that the presence of YPM is limited to the Far East (Japan, Korea and Far-Eastern Russia) [10], and in Japan, Y. pseudotuberculosis harboring YPM were isolated from almost all the fatal cases of breeding monkeys [14]. On the other hand, $Y$. enterocolitica, in particular serotype O3, O5,27, and O9 which are frequent causative agents of yersiniosis and do not harbor HPI, usually cause mild intestinal infection in humans $[4,24]$. Maruyama reported that 10 Crab-eating Macaques (Macaca fascicularis) infected with $Y$. enterocolitica serotype $\mathrm{O} 3$ in experimental infection did not show any noteworthy clinical symptoms, except 3 which showed water diarrhea [20]. Each zoological garden keeps many squirrel monkeys, so even if the squirrel monkeys infected with these low pathogenic strains show the symptoms of yersiniosis, for example mild diarrhea, it is possible that those are passed over, or are not diagnosed as yersiniosis. These results suggested that the squirrel monkeys showing antibodies to Yops have been inapparently or mildly infected with low pathogenic strains of Yersinia, not highly pathogenic strains of Yersinia like YPM producing $Y$. pseudotuberculosis.

The zoological gardens which we investigated kept a number of squirrel monkeys, but did not collect sufficient information on each individual for our research purposes. However, institute $\mathrm{H}$, which is located in the Kyusyu region and keeps the highest number of squirrel monkeys in Japan, individually recognizes all monkeys by electronic microchips. The microchips were implanted into all squirrel monkeys born in the years from 1997 to 2003, so the prevalence of serum antibody to Yops was arranged by age in Institute H. Almost all of the squirrel monkeys which were over 1 year old were positive, while the positive rate of those under 1 year old was only $23.3 \%$ (Table 2 ). These results suggest that the majority of breeding squirrel monkeys in Japan were probably infected by pathogenic Yersinia within one year of birth. As described above, in the present study, many squirrel monkeys that have never shown clinical signs of yersiniosis had the antibody to Yops. It is likely that inapparent infections of low pathogenic Yersinia frequently occur in breeding squirrel monkeys in Japan.

The present study demonstrated that pathogenic Yersinia is highly prevalent among breeding monkeys in Japan. Pathogenic Yersinia is a causal agent of zoonotic disease, and we cannot deny the possibility of human infection from monkeys. Therefore, from the point of view of public health, it is important to develop preventive methods to prevent pathogenic Yersinia infection in monkeys. However, as described above, pathogenic Yersinia strains are widely distributed in wild animals and livestock, so it is possible 
that pathogenic Yersinia is distributed around zoological gardens. Many zoological gardens maintain breeding monkeys not only in indoor cages, but also outdoor cages or enclosures to which wild animals have easy access, so it is difficult to prevent pathogenic Yersinia infection in breeding monkeys even with proper attention to facility maintenance and sanitation, as well as feed hygiene. Therefore, development of an effective vaccine is important for preventing pathogenic Yersinia infection in breeding monkeys.

\section{REFERENCES}

1. Abe, J., Onimaru, M., Matsumoto, S., Noma, S., Baba, K., Ito, Y., Kohsaka, T. and Takeda, T. 1997. Clinical role for a superantigen in Yersinia pseudotuberculosis infection. J. Clin. Invest. 99: 1823-1830.

2. Buhles, W. C., Vanderlip, J. E. Jr., Russell, S. W. and Alexander, N. L. 1981. Yersinia pseudotuberculosis infection: study of an epizootic in squirrel monkeys. J. Clin. Microbiol. 13: 519-525.

3. Butler, T. 1998. Yersiniosis and plague. pp. 281-293. In: Zoonoses (Palmer, S. R., Soulsby, L. and Simpson, D. I. H. eds.), Oxford University Press, Oxford.

4. Carniel, E. 1999. The Yersinia high-pathogenicity island. Int. Microbiol. 2: 161-167.

5. Chatzipanagiotou, S., Ladis, V., Berdousi, H., Palamidou, F., Kourti, E. and Kattamis, C. 1999. Prevalence of yersinia plasmid-encoded outer protein (Yop) class-specific antibodies in multitransfused Greek patients with thalassemic syndromes. Clin. Microbiol. Infect. 5: 67-72.

6. Cornelis, G. R., Boland, A., Boyd, A. P., Geuijen, C., Iriarte, M., Neyt, C., Sory, M. P. and Stainier, I. 1998. The virulence plasmid of Yersinia, an antihost genome. Microbiol. Mol. Biol. Rev. 62: 1315-1352.

7. Fredriksson-Ahomaa, M. and Korkeala, H. 2003. Low occurrence of pathogenic Yersinia enterocolitica in clinical, food, and environmental samples: a methodological problem. Clin. Microbiol. Rev. 16: 220-229.

8. Fredriksson-Ahomaa, M., Naglic, T., Turk, N., Seol, B., Grabarevic, Z., Bata, I., Perkovic, D. and Stolle, A. 2007. Yersiniosis in zoo marmosets (Callitrix jacchuss) caused by Yersinia enterocolitica 4/O:3. Vet. Microbiol. 121: 363-367.

9. Fukushima, H. 2006. Yersinia pseudotuberculosis. pp. 283-316. In: The Prokaryotes, A Handbook on the Biology of Bacteria, volume 6, Proteobacteria (Dworkin, M., Falkow, S., Rosenberg, E., Schleifer, K. H. and Stackebrandt, E. eds.), Springer, New York.

10. Fukushima, H., Matsuda, Y., Seki, R., Tsubokura, M., Takeda, N., Shubin, F. N., Paik, I. K. and Zheng, X. B. 2001. Geographical heterogeneity between Far Eastern and Western countries in prevalence of the virulence plasmid, the superantigen Yersinia pseudotuberculosis-derived mitogen, and the high-pathogenicity island among Yersinia pseudotuberculosis strains. J. Clin. Microbiol. 39: 3541-3547.

11. Hirai, K., Suzuki, Y., Kato, N., Yagami, K., Miyoshi, A. and Mabuchi, Y. 1974. Yersinia pseudotuberculosis infection occurred spontaneously in a group of Patas monkeys (Erythrocebus patas). Jpn. J. Vet. Sci. 36: 351-355.

12. Heesemann, J., Gross, U., Schmidt, N. and Laufs, R. 1986. Immunochemical analysis of plasmid-encoded proteins released by enteropathogenic Yersinia sp. grown in calcium-deficient media. Infect. Immun. 54: 561-567.
13. Heesemann, J., Schröder, J. and Ulrech, M. 1988. Analysis of the class-specific immune response to Yersinia enterocolitica virulence associated antigens in oro-gastrically infected rabbits. Microb. Pathog. 5: 437-447.

14. Iwata, T., Une, Y., Okatani, A. T., Kato, Y., Nakadai, A., Lee, K., Watanabe, M., Taniguchi, T., Elhelaly, A. E., Hirota, Y. and Hayashidani, H. 2008. Virulence characteristics of Yersinia pseudotuberculosis isolated from breeding monkeys in Japan. Vet. Microbiol. 129: 404-409.

15. Kageyama, T., Ogasawara, A., Fukuhara, R., Narita, Y., Miwa, N., Kamanaka, Y., Abe, M., Kumazaki, K., Maeda, N., Suzuki, J., Gotoh, S., Matsubayashi, K., Hashimoto, C., Kato, A. and Matsubayashi, N. 2002. Yersinia pseudotuberculosis infection in breeding monkeys: detection and analysis of strain diversity by PCR. J. Med. Primatol. 31: 129-135.

16. Laemmli, U. K. 1970. Cleavage of structural proteins during the assembly of the head bacteriophage T4. Nature 227: 680-685.

17. MacArthur, J. A. and Wood, M. 1983. Yersiniosis in a breeding unit of Macaca fascicularis (cynomolgus monkeys). Lab. Anim. 17: $151-155$.

18. Mair, N. S. 1973. Yersiniosis in wildlife and its public health implications. J. Wildl. Dis. 9: 64-71.

19. Martinez, R. J. 1983. Plasmid-mediated and temperature-regulated surface properties of Yersinia enterocolitica. Infect. Immun. 41: 921-930.

20. Maruyama, T. 1973. Studies on biological characteristics and pathogenicity of Yersinia enterocolitica. 2. Experimental infection in monkeys. Jpn. J. Bacteriol. 28: 413-421.

21. Olsson, J., Edqvist, P. J., Bröms, J. E., Forsberg, Å, Wolf-Watz, H. and Francis M. S. 2004. The YopD translocator of Yersinia pseudotuberculosis is a multifunctional protein comprised of discrete domains. J. Bacteriol. 186: 4110-4123.

22. Robins-Browne, R. M., Bordun, A. M. and Slee, K. J. 1993. Serological response of sheep to plasmid-encoded proteins of Yersinia species following natural infection with $Y$. enterocolitica and Y. pseudotuberculosis. J. Med. Microbiol. 39: 268-272.

23. Rosenberg, D. P., Lerche, N. W. and Henrickson, R. V. 1980. Yersinia pseudotuberculosis in a group of Macaca fascicularis. J. Am. Vet. Med. Assoc. 177: 818-819.

24. Schiemann, D. A. 1989. Yersinia enterocolitica and Yersinia pseudotuberculosis. pp. 601-672. In: Foodborne Bacterial Pathogens (Doyle, M. P. ed.), Marcel Dekker, New York.

25. Straley, S. C., Skrzypek, E., Plano, G. V. and Bliska, J. B. 1993. Yops of Yersinia spp. pathogenic for humans. Infect. Immun. 61: 3105-3110.

26. Strobel, E., Heesemann, J., Mayer, G., Peters, J., MüllerWeihrich, S. and Emmerling, P. 2000. Bacteriological and serological findings in a further case of transfusion-mediated Yersinia enterocolitica sepsis. J. Clin. Microbiol. 38: 2788-2790.

27. Taffs, L. F. and Dunn, G. 1983. An outbreak of Yersinia pseudotuberculosis infection in a small indoor breeding colony of redbellied (Saguinus labiatus) tamarins. Lab. Anim. 17: 311-320.

28. Tomaso, H., Mooseder, G., Dahouk, S. A., Bartling, C., Scholz, H. C., Strauss, R., Treu, T. M. and Neubauer, H. 2006. Seroprevalence of anti-Yersinia antibodies in healthy Austrians. Eur. J. Epidemiol. 21: 77-81.

29. Torres, B. A., Kominsky, S., Perrin, G. Q., Hobeika, A. C. and Johnson, H. M. 2001. Superantigens: the good, the bad, and the ugly. Exp. Biol. Med. 226: 164-176.

30. Une, Y., Isobe, K., Baba, T., Hayashidani, H. and Nomura, Y. 2003. Yersinia pseudotuberculosis squirrel monkeys. Jpn. J. Zoo. Wildl. Med. 8: 19-26 (in Japanese). 